\title{
SÍNTESIS TRICOMPONENTE DE 5,10-DIHIDROPIRIMIDO[4,5-B] QUINOLEINA-2,4(1H,3H)-DIONA A PARTIR DE ANILINA, ÁCIDO BARBITÚRICO Y BENZALDEHÍDOS AROMÁTICOS SUBSTITUIDOS
}

\author{
SYNTHESIS OF 5,10-DIHYDROPYRIMIDO[4,5-B] QUINOLINE-2,4 \\ (1H, 3H)-DIONE TRICOMPONENT FROM ANILINE, \\ BARBITURIC ACID AND SUBSTITUTED AROMATIC BENZALDEHYDES
}

\author{
Recibido: 05/05/2015
}

Revisado: 28/05/2015

Aceptado: 08/06/2015

\section{Jorge Luis Mendoza Bobadilla', Manuel Alberto Sánchez Pereda', Ronald Fernando Rodríguez Espinoza²}

\section{RESUMEN}

Objetivo: Sintetizar el compuesto 5,10-dihidro pirimido[4,5b] quinoleina-2,4(1H,3H)-diona. Materiales y Métodos: Mediante una reacción multicomponente entre el ácido barbitúrico, la anilina y benzaldehídos aromáticos substituidos, en calentamiento en baño de agua y usando ácido acético como solvente y catalizador. La caracterización estructural de los productos fue realizada mediante sus puntos de fusión y cromatografía de capa fina. Resultados: En la formación de los productos están involucradas las reacciones de condensación Knoevenagel y la adición de Michael, las cuales forman un producto intermedio de cadena abierta; el cual cicla intramolecularmente por la reacción de la función amino nucleofílico de la anilina hacia el carbonilo del ácido barbitúrico, seguido por una deshidratación, conduciendo a la formación de los productos 5,10-dihidropirimido[4,5b]quinoleina-2,4(1 H, 3H)-diona substituidos. Conclusiones: La reacción entre los benzaldehídos aromáticos substituidos, ácido barbitúrico y anilina mediante una reacción multicomponente permitió obtener cuatro productos 5,10-dihidropirimido[4,5-b]quinoleina-2,4$(1 \mathrm{H}, 3 \mathrm{H})$-diona substituidos, con rendimiento entre $56 \%$ y $78 \%$. Y mediante una condensación de Knoevenagel permitió obtener los productos 5-bencilidenpirimidina-2,4,6$(1 \mathrm{H}, 3 \mathrm{H}, 5 \mathrm{H})$-triona substituida, con rendimiento entre $22 \%$ y $36 \%$.

Palabras Clave: Síntesis, condensación, Knoevenagel, Adición, cromatografía de capa fina.

\section{ABSTRACT}

Objective: Make by synthesis the compound of 5,10 dihydropyrimido[4,5-b]quinoline-2,4 (1H, 3H)-dione. Materials and methods: Using a multicomponent reaction between barbituric acid, aniline and substituted aromatic benzaldehydes, in water bath heating and using acetic acid as solvent and catalyst. The structural characterization of the products was made by melting points and thin layer chromatography. Results: In the products formed are involved Knoevenagel condensation and Michael addition reactions, which form an open chain intermediate product; this product; which intramolecularly cyclized by the nucleophilic amino function reaction of the aniline to the carbonyl of the barbituric acid, followed by dehydration, leading to the formation of products 5,10 dihydropyrimido[4,5-b] quinoline-2,4 (1H, 3H)-dione substituted. Conclusions: The reaction between the substituted aromatic benzaldehydes, barbituric acid and aniline using a multicomponent reaction allowed to obtain four products 5,10-dihydropyrimido[4,5-b]quinoline-2,4(1 $\mathrm{H}$, $3 \mathrm{H})$-dione substituted. And by Knoevenagel condensation was obtained 5-bencilidenpyrimidine-2,4,6- $(1 \mathrm{H}, 3 \mathrm{H}, 5 \mathrm{H})$ trione substituted, yield between $22 \%$ and $36 \%$.

Keywords: Synthesis, condensation, Knoevenagel, Addition, thin layer chromatography.

\section{INTRODUCCIÓN}

Las reacciones multicomponentes son procesos que involucran al menos tres componentes diferentes para formar un nuevo producto, que incorpora la mayoría o totalidad de los materiales de partida (Tietze, 2006). Actualmente, la mayor parte de los esfuerzos científicos se han centrado en el desarrollo de procedimientos multicomponentes para preparar diversos compuestos orgánicos. Esto se debe a la simplicidad y economía de éste tipo de reacciones al igual que por la diversidad y complejidad estructural de los compuestos obtenidos (Nenajdenko, 2012).

En el presente trabajo de investigación se describe la preparación de una serie de compuestos 5,10dihidropirimido[4,5-b]quinoleina-2,4(1H,3H)-diona substituidas, 1 , a partir de aldehídos aromáticos 2 , anilina 3 y ácido barbitúrico 4, mediante una reacción multicomponente. Las 5,10-dihidropirimido [4,5b]quinoleina-2,4(1H,3H)-diona son derivados funcionales de la quinoleina, 5 y de la pirimidina, 6, (Carey, 2003).
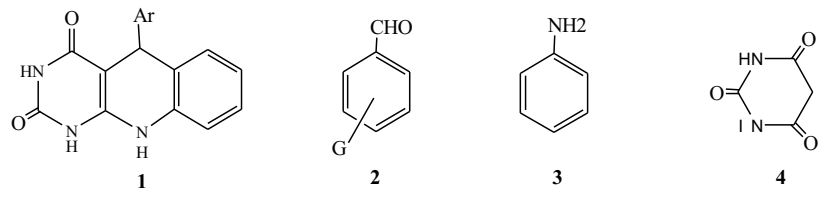
<smiles>c1ccc2ccccc2c1</smiles>

5<smiles>c1cncnc1</smiles>

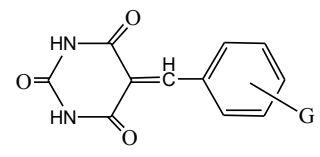

Los aldehídos aromáticos y el ácido barbitúrico interactúan mediante la reacción de Knoevenagel, produciendo un producto intermedio, 7 , posteriormente la anilina, 2, ataca nucleofílicamente al carbono $\beta$ del sistema conjugado de la dicetona insaturada, 7 , mediante la reacción de Adición de Michael, seguido de una deshidratación, conduciendo a la formación de los productos, 1 .

1. Reaccion de Knoevenagel: Es la condensación de aldehídos o cetonas con un grupo metileno activo, en presencia de una base, es conocida con el nombre de reacción de Knoevenagel (Primo, 1994). Las sustancias 1,3-dicetonas con un hidrógeno $\alpha$ reactivo como la dimedona y el ácido barbitúrico, 4, condensan fácilmente con grupos carbonilo de aldehídos o cetonas, en presencia de un catalizador básico (dietilamina, piperidina, etcétera). La reacción da origen a compuestos que se caracterizan por tener el doble enlace carbono-carbono conjugado con los grupos carbonilo, en un sistema $\alpha, \beta$-insaturado (Wade, 2004). Por ejemplo, en la condensación de la acetofenona, 8, con la pentano-1,3-diona, 9, catalizada por piperidina, se forma el derivado de ciclopentiliden-acetofenona 10.<smiles>CC(=O)c1ccccc1</smiles>

8<smiles>O=C1CCC(=O)C1</smiles>

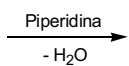

9<smiles>CC(=C1C(=O)CCC1=O)c1ccccc1</smiles>

10
2. Adición de Michael: Se llama así cuando los compuestos con grupos metilénicos activos, dan productos de adición con cetonas y ésteres $\alpha, \beta$-insaturados. La reacción se cataliza con bases, como etóxido de sodio, piperidina, etcétera. En la adición, el carbanión procedente del compuesto metilénico activo se une a la posición $\beta$, mientras que el carbono $\alpha$ del sistema conjugado adiciona un protón, para formar un aducto saturado (Mcmurry, 2001). Así, la adición de acetoacetato de etilo, 12, a la ciclohex-2enona, 11, forma el dicetoéster 13.

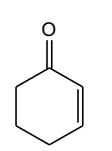

$\left.\right|_{\mathrm{COCH}_{3}} ^{\mathrm{CH}_{2}-\mathrm{COOEt}}$

12

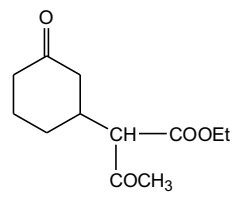

13
3. Quinoleína: Las quinoleínas son compuestos orgánicos heterocíclicos nitrogenados. Se consideran los heterociclos más importantes en los que un anillo de benceno y uno de piridina, 14, se hallan fusionados. Estructuralmente se pueden formular 2 isómeros la quinolina, 5, e isoquinolina 15, (Carey, 2003). Algunos derivados de la quinolina de importancia son la Bezoxiquina, 16, utilizado como desinfectante, la Quinina, 17, utilizada contra la malaria entre otros, (Wade, 2004).
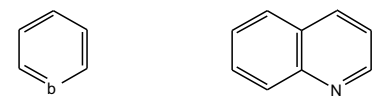

14

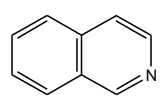

15

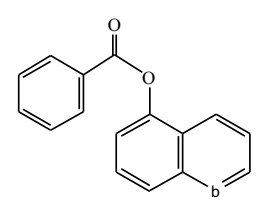

16

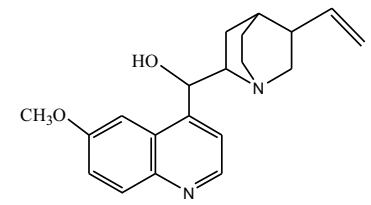

17
4. Pirimidina: Las pirimidinas, 6, son compuestos orgánicos heterocíclicos nitrogenados, similar al benceno y piridina 14, pero que contiene dos átomos de nitrógeno que sustituyen al carbono en las posiciones 1 y 3 . Además, tiene tres derivados muy importantes para la vida como uracilo, 18, timina, 19, y citosina, 20, ya que forman parte de los ácidos nucleicos. La citosina y timina se encuentran en el ADN mientras la citosina y uracilo son las principales pirimidinas presentes en ARN (Carey, 2003). Algunos derivados de la pirimidina de importancia son los alcaloides Teobromina, 21 y la Cafeina, 22, (Mcmurry, 2001).<smiles>O=c1cc[nH]c(=O)[nH]1</smiles>

18<smiles>Cn1cnc2c1c(=O)[nH]c(=O)n2C</smiles>

21<smiles>Cc1c[nH]c(=O)[nH]c1=O</smiles>

19<smiles>Nc1cc[nH]c(=O)n1</smiles>

20<smiles>Cn1c(=O)[nH]c2ncn(C)c2c1=O</smiles>

22

Entre los trabajos que involucran la síntesis de derivados quinolínicos y pirimidínicos, está el realizado por Da-qing S. y colaboradores (2009), en la cual muestran la síntesis de pirimido[4,5-b]quinolina, 23 substituidas vía una reacción tricomponente, mediante una reacción de condensación entre anilinas substituidas, 3 , aldehídos aromáticos 2 , y ácido barbitúrico, 4, en medio acuoso y catalizada con prolina, 24.
$=\gamma_{\mathrm{HN}}^{\mathrm{O}-\mathrm{O}}$

4<smiles>O=Cc1ccc(Cl)cc1</smiles>

2

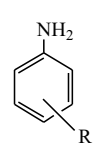

3

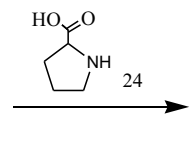

De la misma manera Feng Shi y colaboradores (2011), llevaron a cabo la reacción tricomponente entre aldehídos aromáticos, 2, ácido tetrónico 25, y 5-amino 2metilbenzo[d]tiazol, 26, utilizando ácido acético como solvente y catalizador por irradiación en microondas para obtener derivados de 4-aza-podofilotoxina, 27. Por otro lado, Changsheng y colaboradores (2011), realizaron la sintesis tricomponente de derivados de ciclopenta[b]piranos, 28, mediante una reacción de condensación entre el ciclopentano 1,3-diona, 9, aldehídos aromáticos, 2, y ciano acetato de etilo, 29, con diversos solventes reportando en ácido acético $60 \%$ de rendimiento, libre de solvente $82 \%$ de rendimiento, no obteniendo resultados positivos en medio etanólico.

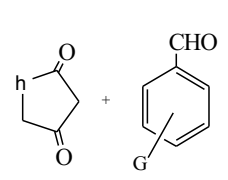

25<smiles>Cc1nc2cc(N)ccc2s1</smiles>

26<smiles></smiles>

H 27 
<smiles>O=Cc1ccc(Cl)c(C=O)c1</smiles><smiles>CCOC(=O)CC</smiles><smiles>[R]C1=C(N)OC2=C(C(=O)CC2)C1[Al]</smiles>

Teniendo en cuenta la importancia que tienen los compuestos con esqueletos quinolínicos y pirimidínicos en sus estructuras, así como la potencial versatilidad de los compuestos con metileno activo como el ácido barbitúrico 4 , en la síntesis de nuevos compuestos heterocíclicos; este trabajo está enfocado en el estudio de la reacción entre ácido barbitúrico 4 , anilina 3 , y aldehídos aromáticos substituidos 2, con el fin de obtener los sistemas pirimido[4,5-b]quinoleina-2,4(1H,3H)-diona, 1, utilizando diferentes condiciones de reacción para evaluar su influencia en la formación de los productos.

El objetivo del trabajo consiste sintetizar el compuesto 5,10-dihidro pirimido[4,5-b] quinoleina2,4(1H,3H)-diona a partir de anilina, ácido barbitúrico y benzaldehídos aromáticos substituidos.

\section{MATERIAL Y MÉTODOS}

Optimización del solvente y temperatura en la obtención de 5-(4-clorofenil) 5,10-dihidro pirimido[4,5b]quinoleina-2,4(1H,3H)-diona.

Se hicieron reaccionar $1 \mathrm{mmol}$ de ácido barbitúrico, 4, como metileno activo, $1 \mathrm{mmol}$ de 4-cloro benzaldehido, 30, 1 mmol de Anilina, 3 , en $10 \mathrm{~mL}$ solvente. Se hirvió a reflujo, por calentamiento convencional. La reacción fue seguida por cromatografía en capa fina (Changsheng, 2011). El 5-(4clorofenil) 5,10-dihidro pirimido[4,5-b]quinoleina$2,4(1 \mathrm{H}, 3 \mathrm{H})$-diona se obtiene mediante el siguiente esquema de reacción:<smiles>O=C1CC(=O)NC(=O)N1</smiles><smiles>O=Cc1ccc(Cl)cc1</smiles>

Cl<smiles>c1ccccc1</smiles>

3

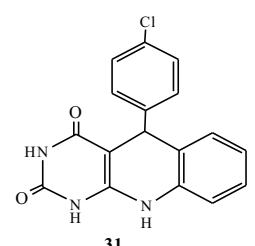

31
Síntesis de pirimido[4,5-b]quinoleina-2,4(1H,3H)diona5,10-dihidro substituidas

Se hicieron reaccionar $1 \mathrm{mmol}$ del respectivo aldehído aromático, $1 \mathrm{mmol}$ de ácido barbitúrico, $1 \mathrm{mmol}$ de Anilina en $10 \mathrm{~mL}$ de ácido acético glacial. Se calentó a $100^{\circ} \mathrm{C}$ en baño de agua por 30 minutos. La reacción fue seguida por cromatografía en capa fina, seguidamente se enfría a temperatura ambiente, se adiciona el doble de agua destilada fría y se deja reposar por un día. Los productos obtenidos se filtraron al vacío y se recristalizaron en etanol diluido.

Síntesis de 5-bencilidenpirimidina-2,4,6-(1H,3H,5H) triona substituida

Los 5-bencilidenpirimidina-2,4,6- $(1 \mathrm{H}, 3 \mathrm{H}, 5 \mathrm{H})$ triona substituida 7 , se obtuvo de acuerdo al siguiente esquema de reacción, en donde los benzaldehídos aromáticos substituidos 2 , reaccionan con ácido barbitúrico 4 , en presencia de catálisis básica.<smiles>O=C1CC(=O)NC(=O)N1</smiles>

4<smiles>O=Cc1ccc(Cl)cc1</smiles>

2<smiles>O=C1NC(=O)C(=C2C=CC(Cl)C=C2)C(=O)N1</smiles>

7
En un balón de $50 \mathrm{~mL}$ se colocó $1 \mathrm{mmol}$ de Acido barbitúrico, $1 \mathrm{mmol}$ de aldehído aromático en $10 \mathrm{~mL}$ de Etanol. Se adicionó 3 gotas de piperidina y se hiervió a reflujo en baño de agua por 30 minutos. La reacción fue seguida por cromatografía en capa fina. Seguidamente se enfrió a temperatura ambiente, se adicionó el doble de agua destilada fría y se deja reposar por un día. Los productos obtenidos se filtraron al vacío y se recristalizaron en etanol diluido.

\section{Punto de fusión}

Los puntos de fusión se determinaron con el equipo digital Mel-Temp, utilizando tubos capilares donde se introduce la muestra para ser leída (Galagovsky, 2002).

\section{Cromatografía de capa fina}

Se utilizaron cromatofolios recubiertos con silica gel y como eluyente una mezcla de bencina de petróleo - acetona en proporciones 1:2, las placas fueron reveladas con vapor de yodo y luz ultravioleta. La constante Rf se midió en términos de la distancia recorrida por la muestra y el eluyente y se calcula de la siguiente manera (Galagovsky, 2002).

$$
R f=\frac{\text { Distancia recorrida por la muestra }}{\text { Distancia recorrida por el eluyente }}
$$

\section{RESULTADOSYDISCUSIÓN}

Los resultados de la optimización del solvente y temperatura en la obtención de 5-(4-clorofenil)-5,10-dihidro pirimido[4,5-b]quinoleina-2,4(1H,3H)-diona, se muestran en la tabla $\mathrm{N}^{\circ} 1$.

Tabla 1. Optimización del solvente y temperatura para la síntesis de 5-(4-clorofenil)-5,10-dihidro pirimido[4,5-b]quinoleina-2,4(1H,3H) diona- substituidas

\begin{tabular}{llccc}
\hline Solvente & $\begin{array}{c}\text { Temperatura } \\
\left({ }^{\circ} \mathbf{C}\right)\end{array}$ & $\begin{array}{c}\text { Tiempo } \\
(\mathbf{m i n})\end{array}$ & $\begin{array}{c}\text { Rendimiento } \\
(\mathbf{\%})\end{array}$ \\
\hline 1 & diclorometano & Reflujo & 60 & trazas \\
2 & etilenglicol & 80 & 60 & trazas \\
3 & polietilenglicol & 80 & 60 & 5 \\
4 & etanol & Reflujo & 60 & 15 \\
5 & etanol / ac. acético proporción 2:1 & 80 & 60 & 25 \\
6 & etanol / ac. acético proporción 1:2 & 80 & 60 & 38 \\
7 & ac. acético & 80 & 60 & trazas \\
8 & ac. acético & ${ }^{\circ}$ T. ambiente & 60 & 78 \\
9 & ac. acético* & 100 & 60 & 7 \\
\hline
\end{tabular}

*Todas las reacciones se llevaron a cabo en la escala de $1 \mathrm{mmol}$ en $10 \mathrm{~mL}$ de solvente bajo calentamiento convencional, excepto la prueba 9 que se llevó a cabo bajo calentamiento en baño de agua. 
Los resultados de la formación de 5,10-dihidro pirimido[4,5-b]quinoleina-2,4(1H,3H) diona substituidas, se muestran en la tabla $\mathrm{N}^{\circ} 2$.

Tabla 2. Formación de 5,10-dihidropirimido[4,5-b] quinoleina-2,4(1H,3H)-diona substituidas

\begin{tabular}{clccc}
\hline Producto Estructura & $\begin{array}{c}\text { Punto de } \\
\text { fusión } \\
\left({ }^{\circ} \mathbf{C}\right)\end{array}$ & Rf & $\begin{array}{c}\text { Rendimiento } \\
\text { (\%) }\end{array}$ \\
\hline 1a & $\mathrm{H}$ & 242 & 0,23 & 56 \\
1b & $p-\mathrm{Cl}$ & 219 & 0,37 & 78 \\
1c & $p-\mathrm{Me}$ & 236 & 0,29 & 72 \\
1d & $p-\mathrm{MeO}$ & 228 & 0,18 & 67 \\
\hline
\end{tabular}

Se sintetizaron 4 diferentes productos pirimidina-2,4,6$(1 \mathrm{H}, 3 \mathrm{H}, 5 \mathrm{H})$ triona substituida cuyas estructuras, puntos de fusión, frentes relativos y rendimientos se muestran en la Tabla $\mathrm{N}^{\circ} 3$.

Tabla 3. Síntesis de pirimidina-2,4,6- $(1 H, 3 H, 5 H)$ -triona substituida

\begin{tabular}{ccccc}
\hline Producto Estructura & $\begin{array}{c}\text { Punto de } \\
\text { fusión } \\
\left({ }^{\circ} \mathbf{C}\right)\end{array}$ & Rf & $\begin{array}{c}\text { Rendimiento } \\
\mathbf{( \% )}\end{array}$ \\
\hline $7 \mathrm{a}$ & $\mathrm{H}$ & 260 & 0,54 & 22 \\
$7 \mathrm{~b}$ & $p-\mathrm{Cl}$ & 238 & 0,55 & 36 \\
$7 \mathrm{c}$ & $p-\mathrm{Me}$ & 266 & 0,41 & 26 \\
$7 \mathrm{~d}$ & $p-\mathrm{MeO}$ & 276 & 0,52 & 25 \\
\hline
\end{tabular}

\section{DISCUSIÓN}

En la tabla $N^{\circ} 1$, los resultados muestran que en presencia de etanol los rendimientos son bajos, generando subproductos uno de ellos y mayoritario el producto 7 , obtenido de la condensación de knoevenagel, entre el ácido barbitúrico y el 4-clorobenzaldehido, el cual disminuye al aumentar el porcentaje de ácido acético. Los mejores resultados se obtienen en ácido acético usado como catalizador y solvente, desapareciendo por completo el producto de la condensación de knoevenagel. Cabe mencionar que, en calentamiento en baño de agua mejora considerablemente el rendimiento del producto, 31 , de $38 \%$ a $78 \%$ en menor tiempo de reacción, debido al calentamiento uniforme proporcionado por el baño de agua.

En la formación de 5,10-dihidro pirimido[4,5b]quinoleina-2,4(1H,3H)-diona substituidos 1 , la reacción transcurre rápidamente, obteniéndose los productos con rendimientos entre $56 \%$ y $78 \%$, dichos rendimientos se consideran aceptables, a pesar de que se optimizaron los rendimientos por recuperación de las aguas madres. Las propiedades de los productos se presentan en la Tabla $\mathrm{N}^{\circ}$ 2. Los valores de $\mathrm{Rf}$ de los 5,10-dihidro pirimido[4,5b]quinoleina-2,4(1H,3H)-diona substituidos registrados en silicagel utilizando el sistema bencina de petróleo / acetona en proporciones 1:2 varían entre 0,18 y 0,37 correspondiendo a estructuras de polaridad elevada. Esta polaridad puede explicarse por la formación de asociaciones por puente de hidrógeno de los nitrógenos amídicos y el adsorbente de la placa cromatográfica, en comparación con el acido barbitúrico cuyo valor de Rf es de
0,05 debido a su menor masa molar y elevada polaridad. Estos compuestos presentan puntos de fusión elevados, varían entre $219{ }^{\circ} \mathrm{C}$ y $242{ }^{\circ} \mathrm{C}$, correspondiendo a compuestos de elevada masa molar, sin embargo, la presencia de los puentes de hidrógeno intramoleculares determinan que estos valores no sean excesivamente elevados.

En la tabla $\mathrm{N}^{\circ} 3$ se muestran las propiedades de los productos obtenidos de la condensación de knoevenagel entre benzaldehídos aromáticos substituidos con ácido barbitúrico, utilizando etanol como solvente y piperidina como catalizador. La reacción transcurre rápidamente obteniéndose productos con rendimientos entre $22 \%$ y $36 \%$. Los productos son de aspecto cristalino, amarillo pálido a intenso. Los valores de Rf de los 5-benciliden pirimidina2,4,6- $(1 \mathrm{H}, 3 \mathrm{H}, 5 \mathrm{H})$ triona, 7 , registrados en silicagel utilizando el sistema bencina de petróleo/acetona en proporciones 1:2 varían entre 0,41y 0,55 correspondiendo a estructuras de polaridad media. Los puntos de fusión de estos compuestos son elevados varían de $238^{\circ} \mathrm{C}$ a $276{ }^{\circ} \mathrm{C}$, correspondiendo a compuestos de elevada masa molar.

En base a lo anterior, se puede concluir que la reacción entre los benzaldehídos aromáticos substituidos, ácido barbitúrico y anilina mediante una reacción multicomponente permitió obtener cuatro productos 5,10 dihidropirimido[4,5-b] quinoleina-2,4-(1H,3H)-diona substituidos, con rendimiento entre $56 \%$ y $78 \%$. Y mediante la condensación de Knoevenagel permitió obtener los productos 5-bencilidenpirimidina-2,4,6- $(1 \mathrm{H}, 3 \mathrm{H}, 5 \mathrm{H})$-triona substituidos, con rendimiento entre $22 \%$ y $36 \%$.

\section{REFERENCIAS BIBLIOGRÁFICAS}

Carey F. (2003). Química Orgánica. Mexico: Mc. Graw Hill.

Changsheng, Y., Bei J., Tuanjie L., Bingbin Q., Xiaodong F., Cuihua W. (2011). Design and an efficient synthesis of natural product-based cyclopenta[b]pyran derivatives with potential bioactivity. Journal of Bioorganic \& Medicinal Chemistry, Letters 21, 599-601.

Da-qing S., Li H., Hong J. (2009). An efficient synthesis of pyrimido[4,5-b]quinoline derivatives via threecomponent reaction in aqueous media. Journal of Bioorganic \& Medicinal Chemistry, Letters.

Feng S., Xiao Z., Ge Z., Shujiang T. (2011). Facile synthesis of new 4-aza-podophyllotoxin analogs via microwaveassisted multi-component reactions and evaluation of their cytotoxic activity. Journal of Bioorganic \& Medicinal Chemistry, Letters 21, 7119-7123.

Galagovsky R. (2002). Fundamentos teóricos prácticos para el laboratorio. Buenos Aires: Editorial Universitaria.

Mcmurry J. (2001). Química Orgánica. Mexico: Thomsom Editores.

Nenajdenko V., Brasche G. (2012). Isocyanide Chemistry Applications in Synthesis and Material Science. Germany: Wiley Vch.

Primo E.D. (1994). Química Orgánica. España: Reverté.

Tietze L., Brasche G. (2006). Domino reactions in Organic Synthesis. Germany: Wiley Vch.

Wade L.G. (2004). Química Orgánica. España: Prentice Hall. 